\title{
Le Théâtre Ruine de Savannah Bay
}

\section{Vassiliki FLENGA}

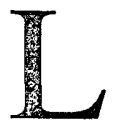

ors d'une interview avec Liliane Papin, Marguerite Duras rend hommage à une grande comédienne qui a toujours tenu les premiers rôles du répertoire durassien: "je viens de faire une pièce pour Madeleine Renaud, spécialement pour elle. Savannah Bay, [. . .]." Dans la version de la pièce représentée au Théâtre du Rond Point, le 27 septembre 1983, Madeleine Renaud monte donc sur le plateau pour jouer le rôle de "Madeleine" dans une œuvre dédiée au théâtre et à son jeu.

Savannah Bay peint le tableau d'une vie éparpillée sur les planches des théâtres lointains, envahie par les rôles joués le long d'une existence. La protagoniste de la pièce, Madeleine, semble avoir effacé de sa mémoire le traumatisme de la perte de sa fille et l'avoir remplacé par des fragments de rôles qu'elle a jadis joués. La ligne de coupure entre la récitation mémorisée d'un rôle et la douleur de l'anamnèse devient ainsi impossible à tracer. D'une part, la mémoire échoue ${ }^{2}$ car, d'après Duras, il s'agit toujours de "la mémoire de l'oubli." D'autre part, cette mémoire oublieuse n'est possible que par l'entremise des Mémoires, terme qui dénote l'écriture d'un rapport sur des événements passés auxquels on s'associe soit comme acteur soit comme témoin. Nous assistons donc à un jeu implacable et sans issue, entre la demande d'un rapport et l'impossibilité de le produire.

Célèbre pour son "expérimentation des formes et saccage des limites,"3 Duras nous a habitué à un théâtre d'écoute, de la non-représentation, où 
l'on assiste à la cérémonie du texte ou encore, à la mise en scène d'une écriture. ${ }^{4}$ Dans Savannah Bay, le ressassement du récit de la pierre blanche envahit et transforme la scène; même si les voix de la pièce sortent de la bouche de ses deux personnages féminins, elles leur sont néanmoins étrangères, venues d'ailleurs. Nous assistons ainsi à la mise en scène de la voix du récit, c'est-à-dire, de la voix narrative. Cette voix impersonnelle, incessante, retentit sur le théâtre vide de Savannah qui n'est plus un lieu du regard, un point de vue sur un événement. Le théâtre de Savannah Bay met en scène des voix qui répètent inlassablement ce qui ne se voit pas: le nom "Savannah," nom insaisissable d'errance et d'égarement qui circule et fuit dans le fond de la scène où se dressent les ruines d'un immense théâtre délaissé. Madeleine ne s'y approche que lentement pendant la représentation, refusant ainsi de l'aborder. A l'écart de ce théâtre qui exhibe sa splendeur ainsi que les fissures du temps, se déroule une comédie désespérée et impossible.

La pièce commence avec la rencontre de deux femmes dans un espace restreint et démuni, indifférent. Faisant contraste à l'austérité de l'aire du jeu, la toile de fond de Savannah Bay se trouve parée d'accessoires splendides. Elle représente la scène imposante d'un théâtre vide. Dans cette partie du plateau, coupée de l'espace de la représentation et désertée par les personnages de la pièce, l'on découvre, derrière deux rideaux, deux colonnes qui encadrent les hauts battants d'une porte dont l'ouverture illuminée par une lumière changeante, représente la mer. Cette embouchure sur l'immensité de la mer, espace qu'on devine seulement et qui reste invisible, produit l'effet d'une scène trouée, vulnérable aux vagues déferlantes.

La représentation de Savannah Bay se trouve donc déplacée, enlevée de l'espace où elle devrait avoir lieu et gardée en otage dans une partie de la scène. Marguerite Duras décrit la scission de la scène de la manière suivante: "Ainsi le décor de Savannah Bay est-il séparé de Savannah Bay, inhabitable par les femmes de Savannah Bay, laissé à lui-même" (93). ${ }^{5}$ Ce décor mythique et sublime, tel un autel sacré, interdit toute approche. Durant la représentation, on assiste à un jeu décalé, se jouant sans scène et tendant désespérément à se rapprocher des ruines superbes, à glisser vers le silence léthéen de la sépulture liquide, à s'abîmer au-delà de la scène.

Les deux femmes de Savannah partagent un récit tragique. La grandmère, Madeleine, et la Jeune Femme sans nom, se voient tous les jours pour se raconter des histoires, incapables d'arrêter, le ressassement d'un récit d'amour mortel, éternel. Il s'agit de narrer sans fin le désir fatal de 
deux amants, leur histoire d'amour impossible qui a donné la mort à la fille de Madeleine, la vie à la Jeune Femme de la pièce. La disparition de la fille de Madeleine constitue ainsi le lien qui sépare les deux femmes, le maillon enlevé de la chaîne laissant derrière un manque immense que les deux survivantes tâchent de combler par le récit.

Pourtant Madeleine, qui a atteint "la splendeur de l'âge," se souvient à peine de son enfant morte ainsi que de l'enfant de cet enfant. Comment raconter une histoire de filiation, quand la mémoire fait défaut, quand Madeleine elle-même ne sait plus qui elle est? Dans l'incipit de la première version de Savannah Bay, l'on trouve l'inscription suivante: "Tu ne sais plus qui tu es, qui tu as été, tu sais que tu as joué, tu ne sais plus ce que tu as joué, ce que tu joues, tu joues, tu sais que tu dois jouer, tu ne sais plus quoi, tu joues." Ces mots tracent le portrait de la protagoniste qui oublie et s'oublie pour répondre à la demande du récit et laisser venir Savannah. La vieille comédienne embarque dans un périple douloureux durant lequel il s'agit de jouer et d'inventer des rôles de/sur la perte, le deuil. L'actrice répète et joue des versions diverses de la même histoire et, ce faisant, elle se trouve constamment traversée par une autre, éclatée dans les bribes de rôles qu'elle a jadis joués, partagée entre Madeleine l'actrice et Madeleine la mère de l'enfant morte. Lors de ce jeu sans issue l'actrice et le personnage qu'elle joue s'imbriquent, les souvenirs d'une noyade indescriptible et sa transcription en rôle mémorisé, répété et récité se relaient ne laissant aucune chance à l'identification. Les rôles joués pendant des années de douleur, équivalent à la répétition d'une mort lente, sans fin: "mourir chaque soir au théâtre. Des mois durant, chaque soir"' (96).

La fille de Savannah retourne voir Madeleine pour exiger d'elle le conte douloureux de sa naissance, un "récit qui porte sur les origines parentales [...]" (50) et qui coïncide avec la noyade tragique de sa mère. ${ }^{6}$ Pour y parvenir, elle fait entendre à Madeleine de vieilles chansons oubliées et lui montre des photographies de famille dans l'espoir de réveiller son passé de comédienne et le souvenir de son enfant perdue. Madeleine ne reconnaît que, "[... ] la photographie d'une femme [qui] a les mains sur le visage, [qui] pleure" (102). Seule la reproduction photographique d'une scène de théâtre suscite sa réponse: "C'est moi. Au théâtre, c'est moi." (102) Propos qui, au lieu de définir une identité, inspirent le doute, murmurant comme un écho, "Je est un[e] autre." Il reste que les efforts de la jeune femme transforment cette dernière en metteur en scène et souffleur d'une douleur aiguë qu'on appelle "Savannah" et qui, "arrive à la vitesse de la lumière. [. . .] disparaît à la vitesse de la lumière. Les mots n'ont plus le temps" (105). Comment accueillir et capter cẹt éclair déferlant, où se mêlent, 
dans un amalgame éblouissant, l'éclat du souvenir et les flashes des photographes? Madeleine n'a qu'à revêtir sa robe rouge de comédienne et se laisser entraîner vers "le lieu incendié de lumière d'un miroir invisible"(105).

Ce miroir, source de lumière dans laquelle se perd l'image de Madeleine et dont on ne voit que le reflet, renvoie à la mer, "illuminée par une lumière variable, soit 'froide,' soit 'brûlante,' soit sombre" (93), selon les indications scéniques. Les deux surfaces miroitantes se réfléchissent et reflètent une force de destruction inouïe: Madeleine, se regardant, se perd et son identité se défait. S'approchant de cette surface étincelante qui consume l'idole, la mère de l'enfant noyée se pare pour jouer son plus grand rôle, pour s'engloutir dans la mer de l'oubli. Évoquée seulement par le jeu de lumière et les voix de deux femmes, la mer, vague paysage de l'au-delà, est encadrée par des accessoires représentant à la fois une scène de théâtre et la maison de la mer/mère d'où Savannah a disparu. Marguerite Duras avait souhaité pour Savannah Bay un décor abstrait, aussi incertain et trompeur que la vie oubliée de la protagoniste:

Je crois que cette maison où elles parlent est la maison de la mer, qu'elle donne sur un espace qui est la mer, que, si on ouvrait la porte ... s'il y avait une grande porte à deux battants, cette porte donnerait droit sur la mer, on verrait l'embouchure du fleuve, là où la petite s'est tuée. Alors, si Madeleine avait laissé les portes ouvertes, toujours, si elle avait perdu la raison, et si elle avait été seule, livrée à elle-même, égarée et folle, si elle n'avait pas joué au théâtre, elle aurait laissé la nature entrer. Et rien ne distinguerait le sol de la maison du sol de l'embouchure. ${ }^{7}$

Le théâtre représenterait donc une sorte de garde-fou, une jetée contre l'abîme des vagues. Mais dans ce paysage maritime incertain, tout bord et toute bordure sont des lignes indécises qu'engouffre l'eau changeante des marées.

C'est sur cette scène d'enlisement que la comédienne amnésique et son assistante entament des scènes et des rôles. Mais qu'est-ce qui est joué au juste? Qu'est-ce qui est récité et répété sur scène? S'agit-il d'un rôle, ou plutôt de fragments de rôles enchevêtrés abandonnés au bon milieu de la répétition, exposant l'échec du récit douloureux d'un deuil impossible, qui, "laissant à l'autre son altérité, en respecte l'éloignement infini, refuse ou se trouve incapable de le prendre en soi comme dans la tombe $[\ldots] . "$ " 
La tentative de produire sur scène le récit d'une histoire d'amour mortel n'aboutit qu'à reconnaître l'impossibilité d'un tel récit. Madeleine trébuche dès qu'elle évoque la pierre d'angle du comte: "On ne peut pas en parler." Difficulté aussitôt remontée car la jeune femme réplique très vite: "On en parle." Le pas entravé vers un récit inénarrable se trouve aussitôt subverti pour aboutir à une narration qui ne raconte que son naufrage dans l'eau salée des pleurs de la mère. Il s'agit d'un mouvement aporétique qui, selon Derrida, "persiste à exécuter ce dont il a montré l'impossibilité." (Mémoires d'Aveugle 133)

Le théâtre de Savannah commence, nous dit Duras, par une tranquille et lente construction de sa scène. Le décor, coupé de l'espace de la représentation, lointain, intervient dans l'élaboration de la pièce intérieure, encadrant le récit entretissé par les deux femmes:

Jeune Femme: C'est une grande pierre blanche, au milieu de la mer.

Madeleine: Plate. Grande comme une salle.

Jeune Femme: Belle comme un palais.

Madeleine: Comme la mer, de la même façon. (108)

L'image que peint cette parole du souvenir et de l'imagination emprunte quelques éléments du décor, le traverse et recule au-delà de l'ouverture de la porte. De cette manière, un passage imperceptible est frayé entre l'avantscène où se joue la pièce et l'arrière-scène, inhabitable pendant la représentation.

La splendeur désertée du bord du plateau, ses accessoires inutiles, constituent un lieu d'attraction qui sous-tend la structure de la pièce. Les deux femmes, décrivant l'espace diégétique de la pièce, espace non-perçu, invisible, évoquent en même temps le décor soustrait de la scène. La pierre blanche, comparée à une salle, puis à un palais, renvoie au décor délaissé, lui ressemblant en beauté, et s'en détache pourtant aussitôt, de la même manière qu'elle s'était jadis séparée de la montagne et avait plongé dans l'immensité de la mer. Cette pierre tombale, sur laquelle est gravée l'épitaphe "Savannah," constitue la pierre d'angle d'une parole sans limite, d'une double parole qui traverse le thêâtre accumulant les débris avant d'atteindre l'ouverture du fond qu'est la mer.

La béance creusée par la lumière sur le mur du fond de la scène ouvre le théâtre à un dehors obscur et informe; du même coup, la ligne de partage entre la scène et le hors-scène s'estompe dans la lumière. L'image de ce théâtre délaissé situé à la bordure de la scène constitue un seuil qu'il faut 
passer pour atteindre la pierre blanche et faire son récit. Pourtant, ce passage est à la fois frayé et impraticable. Dans cette voie entravée, tracée par les voix de deux femmes, a lieu le mouvement piégé du "pas au-delà," mouvement sans mouvement du pas de la marche qui tombe aussitôt en arrêt. Le drame de la diégèse de Savannah, seul drame qui se joue sur scène, reste prisonnier de cette frontière sans garde qu'est le théâtre sur scène. Entre ses deux colonnes imposantes et devant le carré de la porte, s'effectue une ouverture à l'autre, où:

l'autre à la fois est le rapport d'inaccessibilité à l'autre, est l'autre que ce rapport inaccessible institue, est cependant la présence inaccessible de l'autre [. . .] qui se fait rapport et abord dans l'inaccessibilité même de son approche. ${ }^{10}$

Raconter le récit de la pierre blanche, répéter l'histoire de la perte de Savannah, c'est s'oublier pour entrer en rapport avec le neutre, pour passer du "je" au "il" de la voix narrative, ce qui "marque [. . . ] l'intrusion de l'autre -entendu au neutre- dans son étrangeté irréductible, dans sa perversité retorse" (L'Entretien infini 564). Autrement dit, le neutre qui régit le récit "destitue le sujet" et "désapproprie toute possibilité objective" (L'Entretien infini 563). Il s'ensuit que d'une part, le récit n'est raconté par personne et que d'autre part, "les personnages tombent dans un rapport de non-identification avec eux mêmes" (L'Entretien infini 564). Ainsi la voix du récit ne raconte-t-elle qu'à partir de l'oubli, oubli d'ellemême qui invite l'autre absent.

C'est par la formule, "il sans il" que se traduit le neutre blanchotien. La force du sans expose le même "affecté de l'hétérogène absolu qui ne le prive de rien sauf de son identité à soi," selon Derrida (Parages 92). Ce dernier nous explique que l'interposition du sans entre deux termes identiques, ce qui constitue une syntaxe chère à Blanchot, opère de la manière suivante: "le même mot et la même chose paraissent enlevés à euxmêmes, soustraits à leur référence et à leur identité, tout en continuant de se laisser traverser, dans leur vieux corps, vers un tout autre en eux dissimulé" (Parages 90). Suivant une stratégie de division, le sans "remarque le même $X(X$ sans $X)$, sans l'annuler, du tout autre qui l'écarte de lui-même. Absolument, jusqu'à lui faire perdre toute mémoire de soi, tout rapport à soi, [...] toute garde de soi" (Parages 91).

Le sans, formule de soustraction, circule sur la scène de Savannah Bay, qu'il disperse irrémédiablement. Cette pratique de dislocation qui met en relief "la fissure invisible de l'unité,""1 se trouve au cœur du récit, sur la 
surface plate de la pierre massive qu'aucune force de marée ne peut emporter et que l'on évoque croisant d'éléments hétérogènes: "de la mer elle a le grain, le grain de l'eau" (108). Le mouvement périodique de la houle gagne la pierre, rafraîchit l'étendue sèche et se retranche pour laisser la place au soleil brûlant: "presqu'à fleur d'eau, la pierre, quand les bateaux passaient, la houle la recouvrait d'eau frâiche, puis le soleil revenait et en quelques secondes la rendait infernale, de nouveau brûlante" (109). Les rayons transperçants craquent la matière et le bruit du déchirement gagne la scène trouée, se propage pour atteindre le récit et celle qui le raconte péniblement, soutenue par une deuxième voix, perdue, devenue Madeleine sans Madeleine.

La pierre évoque la fille perdue de Madeleine, son souvenir qui émerge et retourne à l'oubli. On nous raconte que le corps mouillé de Savannah laissait son empreinte sur la pierre chaude, trace que le mouvement périodique de la houle effaçait aussitôt. Savannah plonge dans la mer et son image atteint la mère qui se rappelle: "Elle a troué la mer avec son corps. Et elle a disparu dans le trou d'eau. L'eau s'est refermée" (110). Le souvenir devient précis et déstructif:

Madeleine: Je crois que c'était à Montpellier en 1930-1935. Au théâtre de la ville. L'auteur était inconnu. Français, je crois. Pendant ces années-là et les années qui ont suivi, j'étais tous les soirs sur les scènes du théâtre. Partout, dans le monde entier. On aurait pu croire que je jouais différentes choses, mais en fait, je ne jouais que ça, à travers tout ce qu'on croyait que je jouais, je jouais l'histoire de la Pierre blanche. (111)

Souvenir ou aveu qui nous plonge dans l'incertitude, qui retire les repères, et efface les traces comme la mer. Le jeu de Madeleine devient une énigme. Les rôles de Madeleine, la comédienne, s'entrelacent au récit de l'histoire de la mère et de sa fille. Il devient alors impossible de décider si Madeleine est en train de réciter des extraits tirés de son répertoire de comédienne ou bien, si elle se souvient péniblement du suicide de Savannah. Le récit et le jeu d'une séparation douloureuse sont confondus. On passe sans passer de l'un à l'autre, on ne fait que s'approcher de la Pierre blanche sans l'atteindre, sans l'aborder; celle-ci demeure coupée de toute approche et déborde tout abord.

Madeleine évoque et invoque l'inondation, l'engouffrement dans l'épaisseur de la boue: "Je fais ouvrir toutes les portes de la maison, la porte de la Magra, la porte des bateaux, la porte des chambres... que tout 
rentre et tue ... les marécages, la boue, le fleuve" (118). Le jeu ralentit et s'enlise dans la boue oublieuse; les arrêts se succèdent et, durant les pauses, les deux femmes ferment les yeux et demeurent immobiles, perdues dans un repos mortel. Puis, quand la jeune femme pare la comédienne qui se laisse faire, devant le miroir, le jeu reprend, et les deux femmes traversent la scène d'un pas lent:

La Jeune Femme entraîne Madeleine [... . D'abord vers le fond du décor, la porte de la mer, sorte d'autel ouvert sur la mer, sur de la lumière qui s'assombrit ou, au contraire, devient d'une incandescence froide, selon la violence ou la douceur de l'évocation, par les deux femmes, de la jeune fille morte dans la mer chaude de Savannah Bay. Ensuite cette déambulation se fait vers les parages lointains de la scène à proprement parler, vers les rideaux de théâtre et les colonnes qui encadrent la porte. (121)

Cette déambulation met en scène les pas vers le décor de ce théâtre inhabité et inhabitable, lointain et proche à la fois, bordure mouvante qui ne cesse de se transformer en espace de confusion, scène ob-scène toujours admise et à jamais rejetée, séparée de la scène du jeu. On assiste à une sorte de pélérinage vers la tombe de l'enfant morte, vers l'autel liquide et lumineux qui représente à la fois, la scène où le drame pendant des années s'est joué et la maison aux volets fermés d'où Madeleine entendait les voix des amants et ne découvrait que leurs ombres sur les murs. Dans ce théâtre il est impossible de trancher entre le vécu, l'événement et sa représentation, entre ce qui s'est joué sur ses tréteaux et ce qui s'est passé à ses lisières. Savannah Bay représente ainsi la ruine du théâtre, "une ruine qui ne vient pas après l'œuvre mais reste produite dès l'origine, par l'avènement et la structure de l'œuvre" (Mémoires d'aveugle 68). Ce théâtre en ruines ne nous montre rien; la structure dramatique et l'architecture scénique n'admettent pas de relation spéculaire à quelque événement et l'avènement de la scène.

Si par ce passage de deux femmes dans le décor de Savannah Bay, on est tenté de croire que Madeleine ne faisait que jouer la comédie, ne faisait que réciter des rôles de son répertoire, Duras n'hésite point à saper notre recherche désespérée d'un point d'appui. Comment décrire et répéter un amour singulier? "Ensuite, on l'a écrit dans un livre? Madeleine: Je crois. Dans une pièce de théâtre aussi. Et puis ensuite dans un film" (123). Plus tard dans la pièce, on apprend que la Pierre blanche est "une pièce qui n'a été ni jouée ni écrite" (129). Nous sommes face à "un désir de paralysie qui 
n'arrête jamais, qui donne le mouvement et sans mesure" (Parages 73). La structure est labyrinthique, piégée, implacable. Madeleine arrive calmement à une constatation déconcertante:

Rien ... Pour ce qui est de cette pièce-là, rien. Enfin elle n'aura pas été jouée complètement. Mais jamais rien n'est complètement joué précisément au théâtre ... alors ... On croit jouer ça alors qu'on joue ça. [. . .] Tout communique au théâtre, toutes les pièces entre elles mais jamais rien n'est joué vraiment, on fait toujours comme si c'était possible de... (129)

La pièce se joue et s'est jouée sans vraiment l'être; elle se représente enlevée à elle-même. Nous devenons ainsi spectateurs d'encore un autre ravissement implacable.

La mise en scène de la rencontre de deux femmes n'exige le récit de la pierre blanche que pour le subtiliser et le sceller dans le théâtre de la pierre blanche, multiple, incertain, injouable. Savannah Bay raconte et joue un jeu qui échoue: échec de jouer, cauchemar du théâtre, jeu qui s'immobilise, se paralyse et échoue sur le terrain marécageux d'une scène qui tisse une toile où les fils s'entrelacent, où l'on croise une histoire avec son autre. Dans ce thêâtre sans théâtre où il ne reste rien à voir, rien à jouer, "the notion of theater as performance or spectatorship has become obsolete. The mimetic stage is a distant, barely recollectable concept," selon Gabrielle Cody. ${ }^{12}$

La passivité de Madeleine, son attitude désintéressée quant à la redécouverte des événements d'un passé oublié, lointain, laisse transparaître un renoncement inouï. En fait, elle semble frappée d'un certain aveuglement, tourmentée par la volonté aussi bien que par le refus de voir et de savoir. Son regard demeure voilé par le tissu liquide des pleurs de la photographie, ébloui par ce miroir lumineux aui lui ravit le spectre qu'elle tâche désespéremment de ressaisir. Pleurante, elle embarque dans un périple à la fois nécessaire et impossible, durant lequel il ne s'agira plus de voir ni de savoir mais d': "avoir en vue l'imploration plutôt que la vision, adresser la prière, l'amour, la joie, la tristesse plutôt que le regard." 13 En d'autres termes, il ne s'agit plus d'avoir en vue un rapport exact des faits et des dates; Madeleine s'en détourne pour redécouvrir et explorer par la sensibilité et la sensation, plongeant dans l'eau-source des larmes et entraînant à ce naufrage le théâtre, ses spectateurs et ses critiques. 


\section{Notes}

1. Voir, l'ouvrage de Liliane Papin, L'autre scène: Le théâtre de Marguerite Duras (Saratoga, CA: Anma Libri \& Co., 1988) 153.

2. Voir, Suzanne Lamy et André Roy (ed.), Marguerite Duras à Montréal (Montréal: Les éditions Spirale, 1981) 40.

3. Il s'agit d'une formule heureuse de Danielle Bajomée. Voir, l'article de cet auteur, "Veiller sur le sens absent" dans Magazine Littéraire 158 (mars 1980) 32-34.

4. Termes que Liliane Papin propose dans ses études du théâtre durassien, "Théâtres de la non-représentation" paru dans The French Review 64.4 (1991) 667-675 et "Staging Writing or the Ceremony of text in Marguerite Duras" publié dans Modern Drama 34.1 (1991) 128-137.

5. Toutes les citations de Savannah Bay sont tirées de la nouvelle édition qui contient les deux versions de la pièce. La deuxième version fut représentée au Théâtre du Rond Point, avec le décor cré par roberto Plate. Voir, Marguerite Duras, Savannah Bay (Paris: Minuit, 1983)

6. Duras propose ces explications dans les didascalies de la première version de la pièce. Elles sont absentes de la deuxième version.

7. Voir, Roberto Plate, "Le décor de Savannah Bay" Cahiers RenaudBarrault 106 (1983) 7-12.

8. Voir, Jacques Derrida, Mémoires d'aveugle: L'autoportrait et autres ruines (Paris: Éditions de la Réunion des musées nationaux, 1990) 29.

9. Nous faisons, bien entendu, référence à l'ouvrage de Maurice Blanchot intitulé Le pas au-delà (Paris: Gallimard, 1973). Le concept du pas, "à la fois négation et trace ou mouvement de la marche" selon Blanchot, (Voir, L'Écriture du désastre [Paris: Gallimard, 1980] 33) est tracé aussi par Jacques Derrida dans "Pas." (Voir, Parages [Paris: Galilée, 1986] 19-116).

10. Voir, Maurice Blanchot, L' entretien infini (Paris: Gallimard, 1969) 105.

11. Voir, Maurice Blanchot, L'Amitié (Paris: Gallimard, 1971) 190.

12. Voir, son article, "Duras's Theater of Emptied Space and Impossible Performances," paru dans Theater 25.2 (Fall 1994) 65.

13. Dans Mémoires d'aveugle, Jacques Derrida évoque les pleurantes, et des images exorbitantes de larmes dans sa discussion du témoignage. Les larmes qui sont le propre de l'œil pour Derrida, constituent la "ruine" du savoir spéculaire. Voir, Jacques Derrida, Mémoires d'avengle: L'autoportrait et autres ruines (Paris: Éditions de la Réunion des musées nationaux, 1990) 125. 


\section{Ouvrages Cités}

Bajomée, Danielle. "Veiller sur le sens absent." Magazine Littéraire 158 (mars 1980): 32-34.

Blanchot, Maurice. L'entretien infini. Paris: Gallimard, 1969.

-.L'Amitié. Paris: Gallimard, 1971.

—. Le pas au-delà. Paris: Gallimard, 1973.

—. L'Écriture du désastre. Paris: Gallimard, 1980.

Cody, Gabrielle. "Duras's Theater of Emptied Space and Impossible Performances." Theater 25.2 (Fall 1994): 56-66.

Derrida, Jacques. Parages. Paris: Galilée, 1986.

-. Mémoires d' aveugle: L'autoportrait et autres ruines. Paris: Éditions de la Réunion des musées nationaux, 1990.

Duras, Marguerite. Savannah Bay. Paris: Minuit, 1983.

Lamy, Suzanne, et André Roy, ed. Marguerite Duras à Montréal. Montréal: Les éditions Spirale, 1981.

Papin, Liliane. L'autre scène: Le théâtre de Marguerite Duras. Saratoga, CA: Anma Libri \& Co., 1988.

—. "Théâtres de la non-représentation."The French Review 64.4 (1991): 667-675.

-. "Staging Writing or the Ceremony of text in Marguerite Duras." Modern Drama 34.1 (1991): 128-137.

Plate, Roberto. "Le décor de Savannah Bay." Cahiers Renaud-Barrault 106(1983): 7-12. 